\title{
NONLINEAR STABILITY OF MULTISTEP MULTIDERIVATIVE METHODS
}

\author{
SHOUFU LI AND BAOGEN RUAN
}

\begin{abstract}
In this paper we examine nonlinear stability of multistep multiderivative methods for initial value problems of a class $K_{\varphi}$ in a Banach space. Stability criteria are established which extend results of $R$. Vanselow to this class of methods.
\end{abstract}

\section{INTRODUCTION}

Since 1975, theories of nonlinear stability of numerical methods for ordinary differential equations have been gradually developed. However, in most papers the studies are restricted within the limits of finite-dimensional Euclidean spaces (cf. [1-5]). In 1979, Nevanlinna and Liniger [11] were among the first to discuss the stability of one-leg methods for nonlinear problems in a Banach space. In 1983, Vanselow [12] analyzed the stability of linear multistep methods for nonlinear problems of the classes $K 1, K 2 \lambda^{*}$, and $K 3 \mu$ in Banach spaces. Recently, Li [8] introduced the classes of nonlinear problems $K\left(\mu, \lambda^{*}\right)$ and $K\left(\mu, \lambda^{*}, \delta\right)$ which unify and extend the classes of problems and the results in [12]. In a further development along similar lines, Li [9] investigated the nonlinear stability of explicit and diagonally implicit Runge-Kutta methods. Furthermore, $\mathrm{Li}[10]$ made a modification to the class $K(\mu, 0,0)$ to deal with the nonlinear stability of multistep methods with first-order total derivative of the right side of the differential equation. In the present paper these studies are extended to multistep methods with higher derivatives, and the results of [10] and some of [12] will be recovered as special cases.

\section{Class of model problems}

Let $X$ denote a real Banach space with the norm $\|\cdot\|, D$ an infinite subset of $X$, and $f:[0,+\infty) \times D \rightarrow X$ a given sufficiently smooth mapping. Consider the initial value problem (or IVP for short)

$$
\begin{gathered}
y^{\prime}(t)=f(t, y(t)), \quad t \geq 0, \\
y(0)=y_{0}, \quad y_{0} \in D .
\end{gathered}
$$

Received November 4, 1988; revised July 11, 1989.

1980 Mathematics Subject Classification (1985 Revision). Primary 65L20; Secondary 65L05.

Project supported by National Natural Sciences Foundation of China. 
Throughout this paper, the assumption is made that the IVP (2.1)-(2.2) can be solved uniquely.

For any $u, v \in D, t \geq 0$, using the mapping $f$, we define a nonnegative function

$$
H_{u, v, t, f}(\xi)=\left\|u-v+\sum_{q=1}^{p}(-1)^{q} \xi_{q}\left[f^{(q-1)}(t, u)-f^{(q-1)}(t, v)\right]\right\|,
$$

where $\xi=\left(\xi_{1}, \xi_{2}, \ldots, \xi_{p}\right) \in \mathbb{R}^{p}, p$ is a positive integer, and the functions $f^{(q)}$ are defined recursively by

$$
\begin{aligned}
f^{(0)}(t, w) & =f(t, w), \\
f^{(q+1)}(t, w) & =\frac{\partial f^{(q)}(t, w)}{\partial t}+\frac{\partial f^{(q)}(t, w)}{\partial y} f(t, w) .
\end{aligned}
$$

In the special case where $X$ is a (complex or real) Hilbert space with the inner product $\langle\cdot, \cdot\rangle$ and the corresponding norm $\|\cdot\|,(2.3)$ is equivalent to

$$
\begin{aligned}
H_{u, v, t, f}(\xi)= & \left\{u-v\left\|^{2}+\sum_{q=1}^{p} \xi_{q}^{2}\right\| f^{(q-1)}(t, u)-f^{(q-1)}(t, v) \|^{2}\right. \\
& +2 \sum_{q=1}^{p}(-1)^{q} \xi_{q} \operatorname{Re}\left\langle u-v, f^{(q-1)}(t, u)-f^{(q-1)}(t, v)\right\rangle \\
& +2 \sum_{1 \leq i<j \leq p}(-1)^{i+j} \xi_{i} \xi_{j} \\
& \left.\cdot \operatorname{Re}\left\langle f^{(i-1)}(t, u)-f^{(i-1)}(t, v), f^{(j-1)}(t, u)-f^{(j-1)}(t, v)\right\rangle\right\}^{1 / 2} .
\end{aligned}
$$

For convenience, the $H_{u, v, t, f}(\xi)$ will be denoted by $H(\xi)$.

Definition 1. Let $\varphi: \mathbb{R}_{+}^{p} \rightarrow \mathbb{R}_{+}$denote a nonnegative function with the property:

$$
\gamma \varphi(\xi)=\varphi(\gamma \xi) \quad \forall \gamma \in \mathbb{R}_{+}, \xi=\left(\xi_{1}, \xi_{2}, \ldots, \xi_{p}\right) \in \mathbb{R}_{+}^{p} .
$$

Here, $\mathbb{R}_{+}=\{x \in \mathbb{R} \mid x \geq 0\}$. The class of all IVP's (2.1)-(2.2) with $f$ satisfying

$$
\left\{\begin{array}{l}
(\mathrm{I})[1+\varphi(\xi)] H(0) \leq H(\xi) \quad \forall \xi \in \mathbb{R}_{+}^{p}, u, v \in D, t \geq 0 ; \\
\text { (II) for any } \xi=\left(\xi_{1}, \xi_{2}, \ldots, \xi_{p}\right), \tilde{\xi}=\left(\tilde{\xi}_{1}, \tilde{\xi}_{2}, \ldots, \tilde{\xi}_{p}\right) \in \mathbb{R}^{p}, \\
\quad \text { if }\left|\xi_{q}\right| \leq \tilde{\xi}_{q} \text { with } q=1,2, \ldots, p, \\
\text { then } H(\xi) \leq H(\tilde{\xi}) \quad \forall u, v \in D, t \geq 0
\end{array}\right.
$$

is denoted by $K_{\varphi}^{(p)}$, or, if no confusion can arise, simply by $K_{\varphi}$.

Proposition 1. If the IVP (2.1)-(2.2) belongs to the class $K_{\varphi}$, then for any $\xi=$ $\left(\xi_{1}, \xi_{2}, \ldots, \xi_{p}\right) \in \mathbb{R}_{+}^{p}$ and $\delta \in[0,1]$ there holds

$$
H(\delta \xi) \leq \frac{1+\delta \varphi(\xi)}{1+\varphi(\xi)} H(\xi), \quad u, v \in D, t \geq 0 .
$$


Proof. From Definition 1 we find

$$
\begin{aligned}
H(\delta \xi)= & \|(1-\delta)(u-v) \\
& \quad+\delta\left\{u-v+\sum_{q=1}^{p}(-1)^{q} \xi_{q}\left[f^{(q-1)}(t, u)-f^{(q-1)}(t, v)\right]\right\} \| \\
\leq & (1-\delta) H(0)+\delta H(\xi) \leq \frac{1-\delta}{1+\varphi(\xi)} H(\xi)+\delta H(\xi)=\frac{1+\delta \varphi(\xi)}{1+\varphi(\xi)} H(\xi) .
\end{aligned}
$$

Proposition 2. If $X$ is a (complex or real) Hilbert space, then condition (2.6) implies that

(I) $\quad(-1)^{q-1} \operatorname{Re}\left\langle u-v, f^{(q-1)}(t, u)-f^{(q-1)}(t, v)\right\rangle$

$$
\begin{gathered}
\leq-\varphi\left(e_{q}\right)\|u-v\|^{2}, \quad q=1,2, \ldots, p, u, v \in D, t \geq 0 ; \\
\text { (II) } \quad(-1)^{i+j-1} \operatorname{Re}\left\langle f^{(i-1)}(t, u)-f^{(i-1)}(t, v), f^{(j-1)}(t, u)-f^{(j-1)}(t, v)\right\rangle \\
\leq 0, \quad 1 \leq i, j \leq p, u, v \in D, t \geq 0 ;
\end{gathered}
$$

here, $e_{q}=(0, \ldots, 0,1,0, \ldots, 0)$, with the qth component equal to 1 and the others zero. In addition, if the further assumption is made that the function $\varphi$ satisfies

$$
\varphi^{2}(\xi) \leq \sum_{q=1}^{p} \xi_{q}^{2} \varphi^{2}\left(e_{q}\right) \quad \forall \xi=\left(\xi_{1}, \xi_{2}, \ldots, \xi_{p}\right) \in \mathbb{R}_{+}^{p}
$$

(e.g., $\varphi \equiv 0)$ then condition (2.6) is equivalent to (2.7).

Proof. First suppose condition (2.6) is satisfied; we prove (2.7). Put $x>0$ and choose $\xi=x e_{q}$ in (2.6)(I), so that

$$
\begin{aligned}
{\left[\varphi\left(e_{q}\right)+\frac{x}{2} \varphi^{2}\left(e_{q}\right)\right]\|u-v\|^{2} \leq } & \frac{x}{2}\left\|f^{(q-1)}(t, u)-f^{(q-1)}(t, v)\right\|^{2} \\
& +(-1)^{q} \operatorname{Re}\left\langle u-v, f^{(q-1)}(t, u)-f^{(q-1)}(t, v)\right\rangle .
\end{aligned}
$$

Letting $x \rightarrow+0$ we get $(2.7)(\mathrm{I})$. Further, it follows from (2.6)(II) that

$$
H\left(x e_{i}-e_{j}\right) \leq H\left(x e_{i}+e_{j}\right), \quad i, j=1,2, \ldots, p .
$$

Hence we have

$$
\begin{aligned}
&-\operatorname{Re}\left\langle\frac{1}{x}(u-v)+(-1)^{i}\left[f^{(i-1)}(t, u)-f^{(i-1)}(t, v)\right],\right. \\
&\left.(-1)^{j}\left[f^{(j-1)}(t, u)-f^{(j-1)}(t, v)\right]\right\rangle \leq 0 .
\end{aligned}
$$

Letting $x \rightarrow+\infty$ yields $(2.7)$ (II).

We now prove (2.6) on the assumption that (2.7) and (2.8) are satisfied. Note that $(2.7)(\mathrm{I})$ leads to

$$
\varphi^{2}\left(e_{q}\right)\|u-v\|^{2} \leq\left\|f^{(q-1)}(t, u)-f^{(q-1)}(t, v)\right\|^{2}, \quad q=1,2, \ldots, p,
$$


and therefore we find

$$
\begin{array}{r}
\left(\sum_{q=1}^{p} \xi_{q}^{2} \varphi^{2}\left(e_{q}\right)\right)\|u-v\|^{2} \leq \sum_{q=1}^{p} \xi_{q}^{2}\left\|f^{(q-1)}(t, u)-f^{(q-1)}(t, v)\right\|^{2} \\
\forall \xi=\left(\xi_{1}, \xi_{2}, \ldots, \xi_{p}\right) \in \mathbb{R}_{+}^{p}, u, v \in D, t \geq 0 .
\end{array}
$$

A combination of (2.4), (2.7), (2.8), and (2.9) yields (2.6)(I) and (2.6)(II), and this completes the proof.

Proposition 3. Suppose the IVP (2.1)-(2.2) belongs to the class $K_{\varphi}$. Then for any two solutions $y(t)$ and $z(t)$ of the differential equation (2.1) the following is true:

$$
\begin{gathered}
\left\|y\left(t_{2}\right)-z\left(t_{2}\right)\right\| \leq \exp \left(\left(t_{1}-t_{2}\right) \varphi\left(e_{1}\right)\right)\left\|y\left(t_{1}\right)-z\left(t_{1}\right)\right\|, \quad t_{2} \geq t_{1} \geq 0 \\
\left\|y^{(q)}\left(t_{2}\right)-z^{(q)}\left(t_{2}\right)\right\| \\
\leq\left\|y^{(q)}\left(t_{1}\right)-z^{(q)}\left(t_{1}\right)\right\|, \\
t_{2} \geq t_{1} \geq 0, q=1,2, \ldots, p-1, p \geq 2
\end{gathered}
$$

Proof. We only need to note that the functions

$$
G(t)=\exp \left(t \varphi\left(e_{1}\right)\right)\|y(t)-z(t)\|
$$

and

$$
G_{q}(t)=\left\|y^{(q)}(t)-z^{(q)}(t)\right\|, \quad q=1,2, \ldots, p-1,
$$

are continuous for all $t \geq 0$ and have the left-hand derivatives $D_{-} G(t)$ and $D_{-} G_{q}(t)$ for all $t>0$; it is then easily seen from $(2.6)(\mathrm{I})$, (II) that $D_{-} G(t) \leq 0$ and $D_{-} G_{q}(t) \leq 0$.

Proposition 4. Suppose $\psi:[0,+\infty) \rightarrow \mathbb{C}^{N}$ is a given sufficiently smooth mapping, and $A$ an $N \times N$ matrix satisfying

$$
\mu\left(-(-A)^{q}\right) \leq 0, \quad q=1,2, \ldots, p,
$$

where $\mu(\cdot)$ is the logarithmic matrix norm corresponding to an inner-product norm on $\mathbb{C}^{N}$ (cf. [6]). Then the linear system

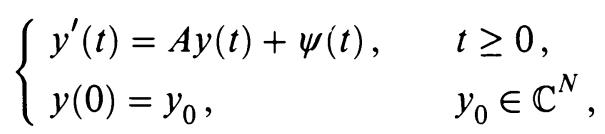

belongs to the class $K_{\varphi}$ with

$$
\varphi(\xi):=-\sum_{q=1}^{p} \xi_{q} \mu\left(-(-A)^{q}\right), \quad \xi=\left(\xi_{1}, \xi_{2}, \ldots, \xi_{p}\right) \in \mathbb{R}_{+}^{p} .
$$

Proof. It is well known that

$$
\left\|(I-A)^{-1}\right\| \leq(1-\mu(A))^{-1} \text { if } \mu(A)<1
$$


and

$$
\mu\left(\sum_{q=1}^{p} \xi_{q} A_{q}\right) \leq \sum_{q=1}^{p} \xi_{q} \mu\left(A_{q}\right) \quad \text { if } \xi_{q} \geq 0, q=1,2, \ldots, p,
$$

where $A_{q}, q=1,2, \ldots, p$, are $N \times N$ matrices and $I$ is the $N \times N$ identity matrix. Hence, under the assumption $(2.12)$ we have $(2.6)(\mathrm{I})$ and one easily shows (2.6)(II) from (2.4).

\section{MAIN RESUlTS AND THEIR PROOFS}

Consider the multistep multiderivative method for the IVP $(2.1)-(2.2)$

$$
\sum_{i=0}^{k} \alpha_{i}\left[y_{n+i}+\sum_{q=1}^{p}(-1)^{q} h^{q} \beta_{i q} f^{(q-1)}\left(t_{n+i}, y_{n+i}\right)\right]=0,
$$

where $k, p$ are positive integers, $h>0$ is a stepsize independent of $n, y_{n+i} \in$ $D$ are approximations to $y\left(t_{n+i}\right), t_{n+i}=(n+i) h$, the coefficients $\alpha_{i}, \beta_{i q}$ are real-valued functions of $h$ (cf. [8] and Examples 4.1 and 4.2 in the present paper), and it is assumed that $\alpha_{k}>0$ and $\sum_{i=0}^{k} \alpha_{i}=0$ for all $h>0$.

For the method (3.1) and any given $h>0$ we define

$$
I_{0}=\{0,1, \ldots, k-1\}, \quad I_{1}=\left\{i \in I_{0} \mid \alpha_{i} \neq 0\right\}, \quad I_{+}=\left\{i \in I_{0} \mid \alpha_{i}>0\right\} .
$$

Note that because of $\alpha_{k}>0$ and $\sum_{i=0}^{k} \alpha_{i}=0$, the set $I_{1}$ is nonempty.

Let $\left\{y_{n}\right\}$ and $\left\{z_{n}\right\} \quad\left(y_{n}, z_{n} \in D\right)$ be two approximation sequences which satisfy (3.1) with different initial conditions. We now introduce some notation:

$$
\begin{aligned}
H_{n}(\xi) & =H_{y_{n}, z_{n}, t_{n}, f}(\xi), \quad w_{n}=y_{n}-z_{n}, \\
F_{n}^{(q-1)} & =f^{(q-1)}\left(t_{n}, y_{n}\right)-f^{(q-1)}\left(t_{n}, z_{n}\right), \quad q=1,2, \ldots, p, \\
A & =A(h)=\sum_{i \in I_{1}}\left|\alpha_{i}\right| /\left|\alpha_{k}\right|=1+2 \sum_{i \in I_{+}} \alpha_{i} / \alpha_{k}, \\
\beta_{i}^{(h)} & =\left(h \beta_{i 1}, h^{2} \beta_{i 2}, \ldots, h^{p} \beta_{i p}\right), \quad i=0,1, \ldots, k .
\end{aligned}
$$

By (3.1) we have at once

$$
\begin{aligned}
w_{n+k} & +\sum_{q=1}^{p}(-1)^{q} h^{q} \beta_{k q} F_{n+k}^{(q-1)} \\
& =-\sum_{i \in I_{1}}\left(\alpha_{i} / \alpha_{k}\right)\left[w_{n+i}+\sum_{q=1}^{p}(-1)^{q} h^{q} \beta_{i q} F_{n+i}^{(q-1)}\right] .
\end{aligned}
$$

Theorem 1. Suppose the IVP (2.1)-(2.2) belongs to the class $K_{\varphi}$ and the set

$$
\begin{aligned}
N_{\varphi}=\left\{h>0\left|\max _{i \in I_{1}}\right| \beta_{i q} \mid \leq \beta_{k q},\right. & q=1,2, \ldots p \\
& \left.\left(1-A r_{0}\right) \varphi\left(\beta_{k}^{(h)}\right) \geq A-1\right\}
\end{aligned}
$$


is nonempty. Then for all $h \in N_{\varphi}$ and $r \in\left[r_{0}, 1\right]$ the generalized contractivity inequalities

$$
\begin{aligned}
{\left[1+r \varphi\left(\beta_{k}^{(h)}\right)\right]\left\|w_{n+k}\right\| } & \leq\left\|w_{n+k}+r \sum_{q=1}^{p}(-1)^{q} h^{q} \beta_{k q} F_{n+k}^{(q-1)}\right\| \\
& \leq C_{h} \max _{i \in I_{0}}\left\|w_{n+i}+r \sum_{q=1}^{p}(-1)^{q} h^{q} \beta_{k q} F_{n+i}^{(q-1)}\right\|,
\end{aligned}
$$

$n=0,1,2, \ldots$, are satisfied. Here,

$$
\left\{\begin{array}{l}
C_{h}=\frac{A\left[1+r_{0} \varphi\left(\beta_{k}^{(h)}\right)\right]}{1+\varphi\left(\beta_{k}^{(h)}\right)} \leq 1, \\
r_{0}=\max \left\{r_{1}, r_{2}, \ldots, r_{p}\right\}, \\
r_{q}=\left\{\begin{array}{ll}
\max _{i \in I_{1}}\left|\beta_{i q}\right| / \beta_{k q} & \text { for } \beta_{k q}>0, \\
0 & \text { for } \beta_{k q}=0,
\end{array} \quad q=1,2, \ldots, p .\right.
\end{array}\right.
$$

Proof. For $h \in N_{\varphi}$ it is easy to see that there exists $r$ meeting the requirements of this theorem and certainly $0<C_{h} \leq 1$. With Definition 1 and Proposition 1 it is obvious that

$$
\left[1+r \varphi\left(\beta_{k}^{(h)}\right)\right]\left\|w_{n+k}\right\| \leq H_{n+k}\left(r \beta_{k}^{(h)}\right) \leq \frac{1+r \varphi\left(\beta_{k}^{(h)}\right)}{1+\varphi\left(\beta_{k}^{(h)}\right)} H_{n+k}\left(\beta_{k}^{(h)}\right) .
$$

Note that (3.3) yields

$$
H_{n+k}\left(\beta_{k}^{(h)}\right) \leq \sum_{i \in I_{1}}\left(\left|\alpha_{i}\right| / \alpha_{k}\right) H_{n+i}\left(\beta_{i}^{(h)}\right) .
$$

On the assumption that $\left|\beta_{i q}\right| \leq r_{0} \beta_{k q}, i \in I_{1}, q=1,2, \ldots, p$, we obtain

$$
H_{n+i}\left(\beta_{i}^{(h)}\right) \leq H_{n+i}\left(r_{0} \beta_{k}^{(h)}\right) \leq \frac{1+r_{0} \varphi\left(\beta_{k}^{(h)}\right)}{1+r \varphi\left(\beta_{k}^{(h)}\right)} H_{n+i}\left(r \beta_{k}^{(h)}\right) .
$$

A combination of (3.7), (3.8), and (3.9) leads to

$$
\begin{aligned}
{\left[1+r \varphi\left(\beta_{k}^{(h)}\right)\right]\left\|w_{n+k}\right\| } & \leq H_{n+k}\left(r \beta_{k}^{(h)}\right) \\
& \leq \frac{1+r \varphi\left(\beta_{k}^{(h)}\right)}{1+\varphi\left(\beta_{k}^{(h)}\right)} \cdot \frac{1+r_{0} \varphi\left(\beta_{k}^{(h)}\right)}{1+r \varphi\left(\beta_{k}^{(h)}\right)} \sum_{i \in I_{1}}\left(\left|\alpha_{i}\right| / \alpha_{k}\right) H_{n+i}\left(r \beta_{k}^{(h)}\right) \\
& \leq \frac{A\left[1+r_{0} \varphi\left(\beta_{k}^{(h)}\right)\right]}{1+\varphi\left(\beta_{k}^{(h)}\right)} \max _{i \in I_{0}} H_{n+i}\left(r \beta_{k}^{(h)}\right),
\end{aligned}
$$

which completes the proof.

Corollary 1. Suppose the IVP (2.1)-(2.2) belongs to the class $K_{\varphi}$ and the set

$$
\tilde{N}_{\varphi}=\left\{h>0\left|\max _{i \in I_{1}}\right| \beta_{i q} \mid \leq \beta_{k q}, q=1,2, \ldots, p ; \max _{i \in I_{1}} \alpha_{i}<0\right\}
$$


is nonempty. Then for all $h \in \tilde{N}_{\varphi}$ and $r \in\left[r_{0}, 1\right]$ the inequalities (3.5) are satisfied with

$$
C_{h}=\frac{1+r_{0} \varphi\left(\beta_{k}^{(h)}\right)}{1+\varphi\left(\beta_{k}^{(h)}\right)} .
$$

Proof. The proof is quite easy; we only need to note that in this case we have $\widetilde{N}_{\varphi} \subset N_{\varphi}$ and $A=1$.

Corollary 2. If in the method (3.1) all coefficients $\alpha_{i}, \beta_{i q}$ are independent of $h$ and satisfy

$$
\begin{cases}\alpha_{i} \leq 0 & \text { for } i \in I_{0} \\ \left|\beta_{i q}\right| \leq \beta_{k q} & \text { for } i \in I_{1}, q=1,2, \ldots, p\end{cases}
$$

then

(I) for any IVP (2.1)-(2.2) of the class $K_{\varphi}$ and $h>0$ the inequalities (3.5) are satisfied with $C_{h}$ given by (3.11), whenever $r \in\left[r_{0}, 1\right]$;

(II) the method is $A(\pi /(2 p))$-stable.

Proof. Result (I) is derived from Corollary 1; for the proof of result (II), note first that, as an obvious consequence of Proposition 4, for $\theta \in[\pi-\pi /(2 p), \pi+$ $\pi /(2 p)]$ the linear model problem

$$
\begin{cases}y^{\prime}=\lambda y, & \lambda=|\lambda| \exp (i \theta) \in \mathbb{C}, i=\sqrt{-1} \\ y(0)=y_{0}, & y_{0} \in \mathbb{C}\end{cases}
$$

belongs to the class $K_{\varphi}$ with

$$
\varphi(\xi):=\sum_{q=1}^{p}(-1)^{q} \xi_{q} \operatorname{Re}\left(\lambda^{q}\right) ;
$$

then apply result (I).

Remark 1. Inequalities (3.5) imply

$$
\begin{aligned}
\max _{i \in I_{0}}\left\|w_{n k+i}+r \sum_{q=1}^{p}(-1)^{q} h^{q} \beta_{k q} F_{n k+i}^{(q-1)}\right\| \\
\leq C_{h}^{n} \max _{i \in I_{0}}\left\|w_{i}+r \sum_{q=1}^{p}(-1)^{q} h^{q} \beta_{k q} F_{i}^{(q-1)}\right\|, \quad n=0,1,2, \ldots,
\end{aligned}
$$

and

$$
\begin{aligned}
&\left\|w_{n k+j}\right\| \leq \frac{C_{h}^{n}}{1+r \varphi\left(\beta_{k}^{(h)}\right)} \max _{i \in I_{0}}\left\|w_{i}+r \sum_{q=1}^{p}(-1)^{q} h^{q} \beta_{k q} F_{i}^{(q-1)}\right\|, \\
& n=0,1,2, \ldots, j=0,1, \ldots, k-1 .
\end{aligned}
$$

Inequalities (3.14) and (3.15) characterize the stability of the method (3.1). 
Remark 2. For the method (3.1) with $p=1,2$, the results in the present paper coincide with those in the papers [8] and [10], respectively.

\section{EXAMPLES}

Example 4.1. For problems with oscillatory solutions a class of methods based on trigonometric polynomials was developed by Gautschi [7]. One such method is the implicit one-step method

$$
y_{n+1}-y_{n}=\frac{1}{\omega} \tan (\omega h / 2)\left[f\left(t_{n+1}, y_{n+1}\right)+f\left(t_{n}, y_{n}\right)\right]
$$

where $h>0$ is a stepsize independent of $n$ and $\omega>0$ is the known circular frequency of the true solution. The method (4.1) is of trigonometric order 1 and of algebraic order 2. In comparison with (3.1) we get that $k=p=1$, $\alpha_{0}=-1, \alpha_{1}=1, \beta_{0,1}=-(\omega h)^{-1} \tan (\omega h / 2)$, and $\beta_{1,1}=(\omega h)^{-1} \tan (\omega h / 2)$. Thus, it is easily seen from Corollary 1 that for any IVP $(2.1)-(2.2)$ of the class $K_{\varphi}$ in a Banach space, the numerical solutions obtained by the method (4.1) are stable provided that for some nonnegative integer $m$ the stepsize $h$ satisfies $2 m \pi \leq \omega h<(2 m+1) \pi$.

Example 4.2. It is easy to verify that the method

$$
\begin{aligned}
y_{n+1}-y_{n}= & \frac{h}{6}\left[\left(3+\gamma h^{3}\right) f\left(t_{n+1}, y_{n+1}\right)+\left(3-\gamma h^{3}\right) f\left(t_{n}, y_{n}\right)\right] \\
& -\frac{h^{2}}{12}\left[\left(1+\beta h^{2}+\gamma h^{3}\right) f^{\prime}\left(t_{n+1}, y_{n+1}\right)\right. \\
& \left.-\left(1+\beta h^{2}-\gamma h^{3}\right) f^{\prime}\left(t_{n}, y_{n}\right)\right]
\end{aligned}
$$

with the constants $\beta, \gamma \geq 0$ is of order four, and it is easily seen from Corollary 1 that for any IVP $(2.1)-(2.2)$ of the class $K_{\varphi}$ and any stepsize $h>0$, the numerical solutions obtained by this method are stable.

Example 4.3. Consider the method

$$
\begin{aligned}
y_{n+1}-y_{n}= & h\left[(1+\alpha) f\left(t_{n+1}, y_{n+1}\right)-\alpha f\left(t_{n}, y_{n}\right)\right] \\
& -\frac{h^{2}}{2}\left[\left(\alpha+\frac{7}{10}\right) f^{\prime}\left(t_{n+1}, y_{n+1}\right)+\left(\alpha+\frac{3}{10}\right) f^{\prime}\left(t_{n}, y_{n}\right)\right] \\
& +\frac{h^{3}}{12}\left[\left(\alpha+\frac{3}{5}\right) f^{\prime \prime}\left(t_{n+1}, y_{n+1}\right)-\left(\alpha+\frac{2}{5}\right) f^{\prime \prime}\left(t_{n}, y_{n}\right)\right],
\end{aligned}
$$

which is consistent of order at least 5 , and order 6 for $\alpha=-\frac{1}{2}$. It is easy to show that this method is $A$-stable if $\alpha \geq-\frac{1}{2}$. On the other hand, for $\alpha \geq-\frac{1}{2}$ the assumption (3.12) is fulfilled; thus for any IVP (2.1)-(2.2) of the class $K_{\varphi}$ and any stepsize $h>0$ the numerical solutions obtained by the method (4.3) 
are stable and can be bounded by

$$
\begin{aligned}
&\left\|w_{n}\right\| \leq \frac{C_{h}^{n}}{1+r \varphi\left(\beta_{k}^{(h)}\right)} \| w_{0}-r h(1+\alpha) F_{0}^{(0)}+\frac{1}{2} r h^{2}\left(\alpha+\frac{7}{10}\right) F_{0}^{(1)} \\
&-\frac{1}{12} r h^{3}\left(\alpha+\frac{3}{5}\right) F_{0}^{(2)} \|, \\
& r_{0} \leq r \leq 1 .
\end{aligned}
$$

Now it can be seen that the method (4.3) is applicable to both nonlinear stiff IVP's of the class $K_{\varphi}$ and linear stiff systems.

\section{BIBLIOGRAPHY}

1. K. Burrage and J. C. Butcher, Stability criteria for implicit Runge-Kutta methods, SIAM J. Numer. Anal. 16 (1979), 46-57.

2. __ Nonlinear stability of a general class of differential equation methods, BIT 20 (1980), 185-203.

3. K. Burrage, Nonlinear stability of multivalue multiderivative methods, BIT 20 (1980), 316325.

4. J. C. Butcher, A stability property of implicit Runge-Kutta methods, BIT 15 (1975), 358-361.

5. G. Dahlquist, Error analysis for a class of methods for stiff nonlinear initial value problems (Numer. Anal., Dundee 1975), Lecture Notes in Math., vol. 506, Springer-Verlag, Berlin, 1976, pp. 60-74.

6. __ Stability and error bounds in the numerical integration of ordinary differential equations (Doctoral Thesis), Trans. Roy. Inst. Technol. Stockholm 130 (1959).

7. W. Gautschi, Numerical integration of ordinary differential equations based on trigonometric polynomials, Numer. Math. 3 (1961), 381-397.

8. Shoufu Li, Nonlinear stability of a class of multistep methods, Numer. Math. J. Chinese Univ. 9 (1987), 110-118.

9. __ The nonlinear stability of explicit and diagonally implicit Runge-Kutta methods, Math. Numer. Sinica 9 (1987), 419-430.

10. __ Nonlinear stability of a class of multistep multiderivative methods, Natur. Sci. J. Xiangtan Univ. 1 (1987), 1-8.

11. O. Nevanlinna and W. Liniger, Contractive methods for stiff differential equations, BIT 18 (1978), 457-474 and 19 (1979), 53-72.

12. R. Vanselow, Nonlinear stability behaviour of linear multistep methods, BIT 23 (1983), 388-396.

Department of Mathematics, Xiangtan University, 411105 Xiangtan, Hunan Province, People's Republic of China

Department of Mathematics, Jiujiang Teacher's College, Jiangxi Province, People's RePUblic OF China 